\title{
Disease control with prior platinum-based chemotherapy is prognostic for survival in patients with metastatic urothelial cancer treated with atezolizumab in real-world practice
}

\author{
Marina Mencinger ${ }^{1,2}$, Dusan Mangaroski ${ }^{1}$, Urska Bokal ${ }^{1}$ \\ ${ }^{1}$ Institute of Oncology Ljubljana, Ljubljana, Slovenia \\ ${ }^{2}$ Faculty of Medicine, University of Ljubljana, Ljubljana, Slovenia \\ Radiol Oncol 2021; 55(4): 490-498.
}

Received 27 September 2021

Accepted 07 March 2021

Correspondence to: Assist. Marina Mencinger, M.D., Ph.D., Institute of Oncology Ljubljana, Zaloška 2, 1000 Ljubljana, Slovenia. Phone: +386 15879 286; Fax: +386 15879 303; E-mail: mmencinger@onko-i.si

Disclosure: Roche Farmacevtska Druzba d.o.o has funded the statistical analysis for this study. The interpretation of the results and the content of this publication express the author's independent professional opinion and were not influenced by Roche. Marina Mencinger and Urska Bokal received honoraria for consultancy from Roche, all authors received speaker's honoraria from Roche.

\begin{abstract}
Background. Atezolizumab, a programmed-death ligand-1 (PD-L1) inhibitor, is a novel treatment option for patients with metastatic urothelial cancer (mUC). Clinical prognostic factors, survival outcomes, and the safety of patients with mUC treated with atezolizumab, in a real-world setting, were investigated.

Patients and methods. 62 patients with mUC, treated at the Institute of Oncology Ljubljana between May $8^{\text {th }}$ 2018 and Dec 31 $31^{\text {st }} 2019$, were included. Response rates and immune-related adverse events (irAE) were collected. Progression-free survival and overall survival times were assessed using the Kaplan-Meier method. The Cox proportional hazards model was applied to identify the factors affecting survival.

Results. Of 62 patients, five (8.1\%) have not yet been evaluated and $20(32 \%)$ died prior to the first radiographic evaluation. We observed clinical benefit in $19(33 \%)$, objective response in $12(21 \%)$, and complete response in five (9\%) patients. Median overall survival for the whole population was $6.8(95 \% \mathrm{Cl}, 2.6-11.0)$, for platinum-naiive 8.7 (95\% $\mathrm{Cl}$ : 0.8-16.5), and for the platinum-treated group $6.8(95 \% \mathrm{Cl}, 3.7-10)$ months. At the $5.8(0.3-23.1)$ month median follow-up, the median duration of the response was not reached. IrAE occurred in $20(32 \%)$ patients and seven (11\%) of them discontinued the treatment. Multivariate analysis in platinum-treated patients showed that a treatment-free interval of more than six months was prognostic for overall survival (OS).

Conclusions. Responses to atezolizumab led to long disease remission in a subset of our patients. The median OS in our real-world population was compromised by a large percentage of patients with poor ECOG performance status (PS). A treatment-free interval from chemotherapy was associated with the longer survival of platinum-treated patients with mUC receiving further atezolizumab.
\end{abstract}

Key words: PD-L1 inhibitor; urothelial cancer; bladder; atezolizumab; overall survival; immune checkpoint inhibitor; prognostic factors

\section{Introduction}

Metastatic urothelial carcinoma (mUC) is an aggressive malignancy with poor prognosis among urological cancers. ${ }^{1}$ Standard cisplatin-based chemotherapy as a first-line regimen for $\mathrm{mUC}$ remains a challenge for many patients due to numerous comorbidities. ${ }^{2}$ Patients with mUC not eligible for combination therapy with cisplatin commonly receive carboplatin and have an expected medi- 
an overall survival (mOS) of only nine months. ${ }^{3}$ Moreover, there is a lack of evidence for improved outcomes for second-line therapeutic options: vinflunine is approved only in Europe and taxanes or gemcitabine are commonly used in the USA with only modest efficacy. ${ }^{4,5}$

Recently, programmed death-ligand 1 (PD-L1) and programmed death-1 (PD-1) inhibitors have become the new systemic therapies for patients with mUC with disease progression after platinum-based chemotherapy. ${ }^{6-9}$ Two of them, pembrolizumab and atezolizumab, are also approved for the first-line treatment of cisplatin-ineligible patients whose tumors express PD-L1.10,11 Recently, the large III phase study IMVIGOR 130, investigating atezolizumab as a first-line therapy, alone or in combination with platinum-based chemotherapy, revealed a significant improvement in the progression-free survival (PFS) of atezolizumab added to platinum-based chemotherapy versus platinumbased chemotherapy alone. ${ }^{12}$

Prognostic factors (Eastern Cooperative Oncology Group Performance status, ECOG PS; haemoglobin level and liver metastasis) in patients with mUC after the failure of platinum-based chemotherapy have already been identified. ${ }^{13}$ Recently, novel prognostic factors (high $\mathrm{C}$ reactive protein, poor response to previous chemotherapy and poor ECOG PS) for OS in patients with bladder carcinoma receiving second-line chemotherapy have been proposed. ${ }^{14}$

It was shown that PD-L1-positive mUC had a significantly better response to PD-1/PD-L1 targeted treatment. ${ }^{15}$ It is not clear if PD-L1 has a predictive role for survival benefit with atezolizumab in patients with mUC. ${ }^{16}$ Other clinical and biological parameters beyond PD-L1 expression could affect the benefit from an immune checkpoint blockade.

The aim of this retrospective single-centre study was to identify novel clinical prognostic factors for OS in patients with mUC who received atezolizumab as monotherapy in a real-world setting. Furthermore, response rates in different patient populations, efficacy outcomes and the safety of these patients are reported.

\section{Patients and methods}

A retrospective study on patients with mUC who were treated with atezolizumab at the Institute of Oncology, Ljubljana, from May $8^{\text {th }} 2018$ to December $31^{\text {st }} 2019$ was performed. Patients received atezolizumab after the failure of platinum- based chemotherapy or were cisplatin-ineligible as estimated by the treating oncologist. Patients who were cisplatin-ineligible and initiated atezolizum$\mathrm{ab}$ after August $8^{\text {th }} 2018$ were tested for PD-L1.

The study was approved by the Institutional Review Board Committee (No. 478, date of approval 2019 Apr 25) and was carried out according to the Declaration of Helsinki. All necessary consents required by applicable law from any relevant patient whose information is included in the article have been obtained.

Patients with a confirmed histological diagnosis of inoperable mUC received atezolizumab at a dose of $1200 \mathrm{mg}$ every three weeks until discontinuation. The therapy was discontinued because of death, radiographic disease progression, or unacceptable toxic adverse events. The relevant clinical and laboratory data were obtained from patients' data charts: age, sex, ECOG PS, tumor histology type, number and types of prior systemic therapies, surgery, location of metastasis in different organs at the initiation of atezolizumab, number of atezolizumab applications, the date of the first and last chemotherapy/atezolizumab cycle, PD-L1 status, if available, immune-related adverse events (irAE) as recorded by the treating oncologist. Considering chemotherapy exposure, two groups have been predefined: the first group, platinum-treated, consisted of patients that had received chemotherapy either as neoadjuvant, adjuvant, or as a first- or second-line therapy, and the second group, defined as platinum-naïve, had not received any chemotherapy before atezolizumab.

The objective response rate (ORR) was defined as the proportion of patients with a complete or partial response according to the Response Evaluation Criteria in Solid Tumors (RECIST v 1.1) of all patients that had had at least one radiographic evaluation or who had died before the first radiographic evaluation. Response to treatment was evaluated using a computed tomography of chest and abdomen at baseline and then every 3-4 months or at the discretion of the treating oncologist. ORR was computed separately for platinum-treated and chemotherapy-naïve groups, and for patients with $\operatorname{good}(0,1)$ and poor $(2,3)$ ECOG PS.

Clinical benefit (CB) comprised complete, partial response, or stable disease (CR, PR; SD). Duration of the response was defined as the time between the initial response to therapy and subsequent disease progression or death. A treatmentfree interval (TFI) was defined as the time between the last chemotherapy cycle to the first cycle of atezolizumab. 
Median PFS and mOS for the whole group and mOS for the platinum-treated and the platinum-naive cohorts were computed separately. Progression of the disease was defined as radiographic progression according to RECIST 1.1 or death, whichever occurred first. We presumed that patients who died before the first radiographic evaluation had progressive disease as their best response. PFS and OS were estimated using Kaplan-Meier methods, and the differences were evaluated using the logrank test. PFS was defined as the time from the first administration of atezolizumab to radiographic or clinical progression or death. OS time was analyzed from the start of atezolizumab until death from any cause or until the last follow-up examination. Duration of response was defined as the time from response (CR or PR) to progression/death.

$P$ values $<0.05$ were considered significant, and $95 \%$ confidence intervals (CI) were calculated.

IrAE as assessed by the treating oncologist and graded according to the Common Terminology Criteria for Adverse Events (CTCAE) version 5.0 were assembled.

\section{Results}

Sixty two patients with locally advanced or mUC initiated treatment with atezolizumab at the Institute of Oncology Ljubljana between May $8^{\text {th }}$ 2018 and December $31^{\text {st }} 2019$. According to prior exposure to chemotherapy, platinum-treated and platinum-naïve groups were formed. The characteristics for these patient groups are displayed in Table 1. The group of platinum-treated patients had 44 patients with a median age of 65 (48-80), whereas the group of platinum-naïve patients totaled 18 patients with a median age of $75(62-85)$ years. The majority of the patients in both groups were males. Good ECOG PS $(0,1)$ according to the WHO classification was determined in more than half of them ( 26 or $59 \% ; 10$ or $56 \%$ ) in each group. Primary metastatic disease was detected in $23(52 \%)$ of platinum-treated patients and in $14(78 \%)$ of platinum-naïve patients. PD-L1 staining (Ventana SP142 test) was positive according to the published criteria in $12(67 \%)$ patients initiating first-line treatment with atezolizumab; but in more than half $(25,57 \%)$ of platinum-treated patients testing was not performed and PD-L1 status remained unknown (Table 1). All 18 patients with liver metastasis belong to the platinum-naïve group.
TABLE 1. Patients' characteristics

\begin{tabular}{lcc}
\hline & $\begin{array}{c}\text { Platinum-treated } \\
\mathrm{N}=44(\%)\end{array}$ & $\begin{array}{c}\text { Platinum-naïve } \\
\mathrm{N}=18(\%)\end{array}$ \\
\hline $\begin{array}{l}\text { Median age, years (range) } \\
\text { Age } \geq 75 \text { years }\end{array}$ & $65(48-80)$ & $75(62-85)$ \\
\hline Sex & $27(61) / 17(39)$ & $11(61) / 7(39)$ \\
\hline Male/ Female & $26(59) / 18(41)$ & $10(56) / 8(44)$ \\
\hline ECOG PS & $23(52)$ & $14(78)$ \\
\hline $0,1 / 2,3$ & $14(32) / 30(68) / 18(41)$ & $13(72) / 5(28) / 0$ \\
\hline $\begin{array}{l}\text { Primary metastatic disease } \\
\text { Metastatic site } \\
\text { nonvisceral/visceral/hepatic }\end{array}$ & $35(80)$ & $15(83)$ \\
\hline $\begin{array}{l}\text { Pure urothelial histology } \\
\text { PD-L1 status }\end{array}$ & $9(20)$ & $1(5)$ \\
\hline $0-4 \%$ & $10(23)$ & $12(67)$ \\
\hline$\geq 5 \%$ & $25(57)$ & $5(28)$ \\
\hline $\begin{array}{l}\text { Unknown } \\
\text { Treatment modalities before } \\
\text { atezolizumab }\end{array}$ & $28(93) / 2(7)$ & \\
\hline $\begin{array}{l}\text { Perioperative chemotherapy } \\
\text { Surgery }\end{array}$ & $14(32)$ & $10(56)$ \\
\hline $\begin{array}{l}\text { Chemotherapy for metastatic } \\
\text { disease }\end{array}$ & $25(57)$ & \\
$\begin{array}{l}\text { Number of chemotherapy } \\
\text { lines for metastatic disease } \\
\text { before atezolizumab }\end{array}$ & $30(68)$ & \\
\hline $1 / 2$ & & \\
\hline & & \\
\hline
\end{tabular}

ECOG PS = performance status according to WHO classification

Before the initiation of atezolizumab, more than half the patients in each group underwent a cystectomy. Fourteen platinum-treated patients (32\%) received chemotherapy in perioperative, and 30 $(68 \%)$ in a first-line setting. Most, $28(93 \%)$, received only one line of chemotherapy for metastatic disease before commencing atezolizumab.

Among the 62 included, 57 patients had available data for response analysis. A complete response was obtained in five (9\%), a partial response in seven $(12 \%)$, and a clinical benefit in $19(33 \%)$ patients. The majority, 38 (67\%), had progressive disease as their best response as assessed radiographically or/ and clinically. Liver metastases were detected in $16(26 \%)$ patients with available data for response analysis. No complete response was observed among patients with liver metastases (Table 2).

Median PFS was 4.2 (95\% CI, 1.9-6.5) and mOS 6.8 (95\% CI, 2.6-11.0) months (Figure 1). Of the 62 patients included, $18(29 \%)$ were platinum-naïve and had mOS of 8.7 (95\% CI, 0.8-16.5) months 


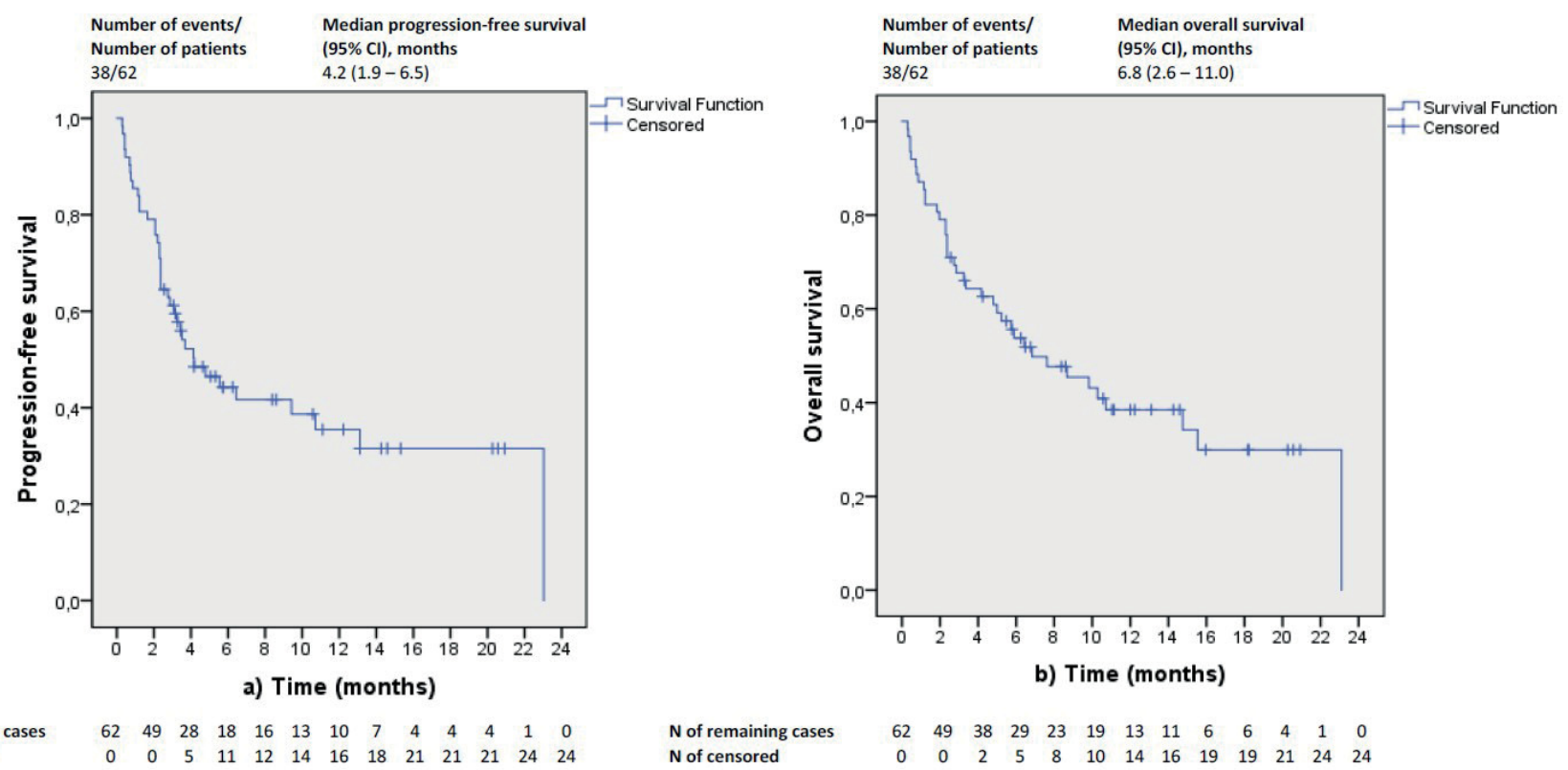

$\begin{array}{llllllllllllll}N \text { of remaining cases } & 0 & 0 & 5 & 11 & 12 & 14 & 16 & 18 & 21 & 21 & 21 & 24 & 24\end{array}$

$\mathrm{N}$ of censored

$\begin{array}{lllllllllllllll}0 & 0 & 2 & 5 & 8 & 10 & 14 & 16 & 19 & 19 & 21 & 24 & 24\end{array}$

FIGURE 1. Kaplan-Meier curves of progression-free survival (A) and overall survival (B) of all study population.

TABLE 2. Responses to treatment with atezolizumab

\begin{tabular}{|c|c|c|c|c|c|c|}
\hline Type of response & $\begin{array}{l}\text { All patients } \\
N=57(\%)\end{array}$ & $\begin{array}{c}\text { Patients with liver } \\
\text { metastases } \\
\mathrm{N}=16(28 \%)\end{array}$ & $\begin{array}{l}\text { Platinum-naive } \\
\qquad \mathrm{N}=17(30 \%)\end{array}$ & $\begin{array}{l}\text { Platinum-treated } \\
\qquad \mathrm{N}=40(70 \%)\end{array}$ & $\begin{array}{c}\text { ECOG PS 0+1 } \\
N=33(58 \%)\end{array}$ & $\begin{array}{c}\text { ECOG PS } 2+3 \\
N=24(42 \%)\end{array}$ \\
\hline$C R$ & $5(9)$ & $0(0)$ & $3(17.5)$ & $2(5)$ & $4(12)$ & $1(4.2)$ \\
\hline$S D$ & $7(12)$ & $4(25)$ & $1(6)$ & $6(15)$ & $6(18)$ & $1(4.2)$ \\
\hline$P D$ & $38(67)$ & $11(69)$ & $10(59)$ & $28(70)$ & $18(55)$ & 20 (83.3) \\
\hline
\end{tabular}

$C R=$ complete response $; P R=$ partial response $; \mathrm{SD}=$ stable disease $; \mathrm{PD}=$ progressive disease

TABLE 3. Univariant and multivariant analysis of prognostic factors (correlation with overall survival)

\begin{tabular}{lccccc}
\hline Subgroup & Univariant HR & $\mathbf{p}$ & Multivariant HR & $\mathbf{p}$ & Reference category \\
\hline Age & $0.861(0.428-1.731)$ & 0.675 & & & \\
\hline ECOG PS & $2.883(1.495-5.559)$ & 0.002 & $3.449(1.358-8.761)$ & 0.009 & 2 or 3 \\
Visceral metastases* & $0.965(0.502-1.853)$ & 0.914 & & Yes & Yes \\
Clinical benefit to previous chemotherapy & $0.319(0.133-0.765)$ & 0.010 & $0.355(0.131-0.961)$ & 0.042 & 0.037 \\
TFI & $0.140(0.032-0.604)$ & 0.008 & $0.113(0.014-0.877)$ & $>6$ months \\
IrAE & $0.566(0.266-1.202)$ & 0.139 & & Yes \\
\hline
\end{tabular}

Clinical benefit (complete, partial response or stable disease); ECOG PS = Eastern Cooperative Oncology Group performance status; HR = hazard ratio; IrAE = immune related adverse events; TFI = treatment free interval; ${ }^{*}=$ defined as presence of non lymph node and non bone metastases

whereas $44(71 \%)$ were platinum-treated with $\mathrm{mOS}$ of 6.8 (95\% CI, 3.7-10) months (Figure 2).

The median duration of response was not estimable at a median follow-up of $5.8(0.3-23.1)$ months (Figure 3).
A proportional Cox hazard model was used to identify prognostic factors associated with OS. Variables that were found to be significant in univariate analysis were selected for further evaluation in a multivariate model. According to uni- 

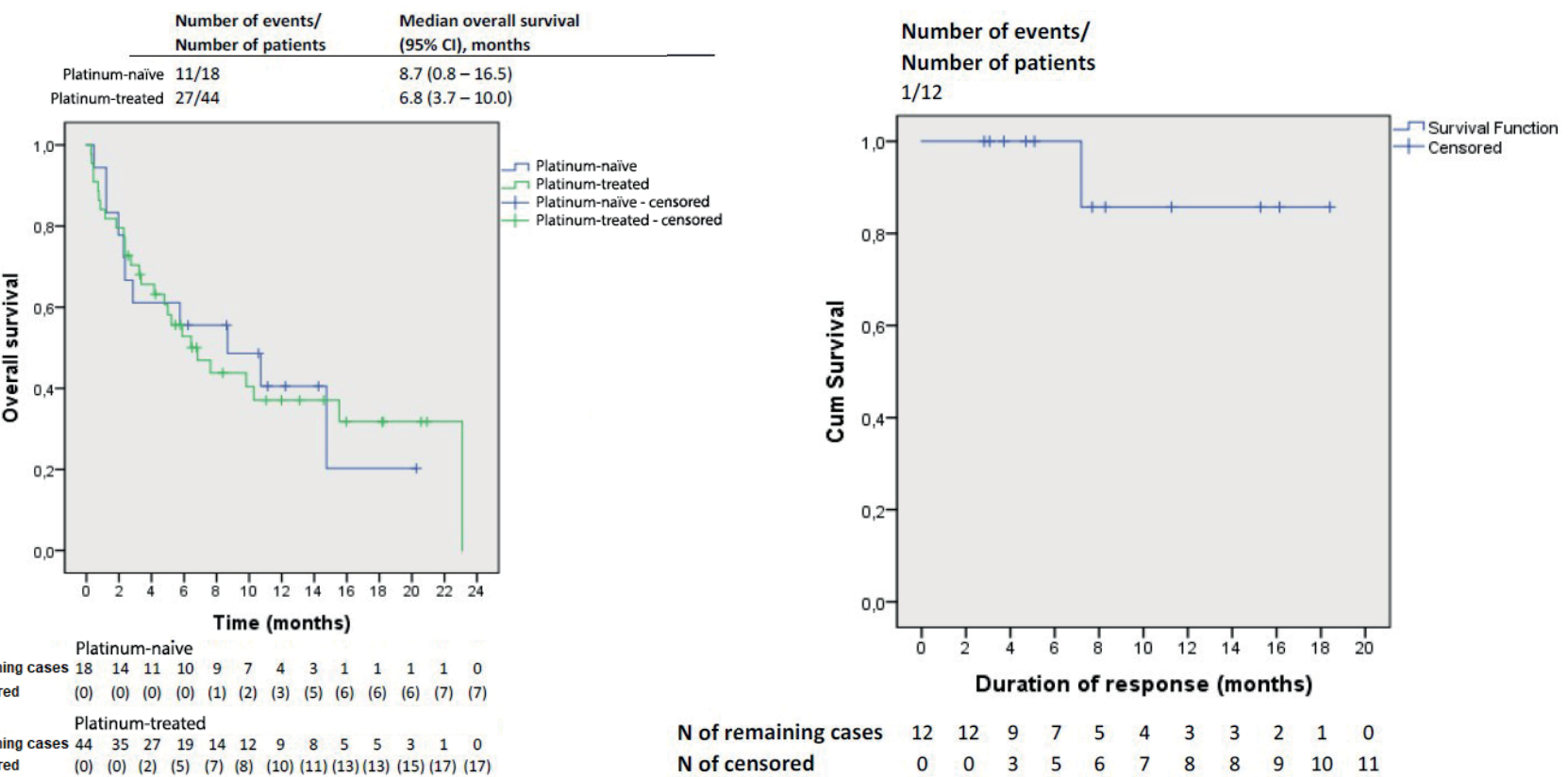

FIGURE 2: Kaplan-Meier curves of overall survival according to the previous exposure to chemotherapy.

TABLE 4. Immune-related adverse events

\begin{tabular}{lc}
\hline IrAE of any grade & $20(32 \%)$ \\
IrAE $\geq$ Grade 3/4 & $7(11 \%)$ \\
Systemic corticosteroid use & $5(8 \%)$ \\
Atezolizumab discontinuation due to IrAE & $7(11 \%)$ \\
\hline
\end{tabular}

IrAE = immune related adverse events

variate analysis the negative prognostic factors for overall survival were ECOG PS 2-3, no CB to prior chemotherapy, and TFI less than six months. Only TFI of less than six months impacted poor survival in multivariate analysis. Age, non-visceral disease (defined as metastases in lymph nodes and/ or bones only) and the presence of irAE were not proven to be statistically significant in univariate analysis (Table 3).

\section{Safety}

The type and severity of irAE were collected. Twenty patients $(32 \%)$ suffered from irAE, and seven $(11 \%)$ of them had grade $3-4$ irAE as estimated by the treating oncologist and according to the CTCAE v. 5.0. All seven (11\%) patients with 3-4 grade toxicity discontinued the therapy. Five $(8 \%)$ patients were treated with systemic corticosteroids and one patient received only topical corticosteroid therapy (Table 4).
The most common irAE was skin toxicity (eight, $40 \%$ of the affected patients), followed by hepatopathy (four, 20\%), arthritis, and central nervous toxicity (two, 10\%). Hypothyroidism, nephropathy, low platelet count, and gastrointestinal adverse events were annotated in one patient each.

\section{Discussion}

The results of our study show that atezolizumab induced long-lasting responses in a subset of patients with mUC. The median duration of response was not reached at a median follow-up of 5.8 months (0.3-23.1), similarly to what was observed in other prospective trials studying the efficacy of atezolizumab in patients with mUC. $8,12,17,18$

The ORR in our platinum-naive and platinumtreated groups were comparable to ORR of similar patient groups in prospective trials; however, this did not translate to the same extent of survival benefits as seen in prospective trials, where the selection of patients is stricter.

Notably, a higher proportion of platinum-naive patients achieved OR (35\%) compared to the platinum-treated cohort $(15 \%)$. This is, however, in line with the reported ORR of similar patient groups in two other trials. In the nonrandomized IMVIGOR 210 trial, the ORR in cohort 1 (cisplatinineligible) was $23 \%$ and in cohort 2 (platinum- 
treated) $15 \% .^{10,17}$ Similarly, in a larger phase III clinical trial IMVIGOR 211, which assigned 931 platinum-treated patients, the ORR was only $13.4 \%$ in the ITT population regardless of PDL-1 testing. ${ }^{8}$ Although PDL-1 testing has not been performed in all our patients, it was true that the platinum-naive cohort was enriched with PD-L1 positive patients (Table 1). Namely, during our study the label for atezolizumab has been updated by the US Food and Drug Administration (FDA) and the European Medicines Agency (EMA), which restricted the use of atezolizumab to cisplatin-ineligible patients who have a positive PD-L1 score. This selection of patients for first-line atezolizumab therapy at least partially explains the higher ORR in our platinumnaive group. Our platinum-naïve cohort corresponded to the cisplatin-ineligible population as defined by the Galsky criteria which, among others, include poor ECOG PS commonly encountered among these patients. ${ }^{3}$ The predictive value of PD-L1 for atezolizumab in patients with urothelial cancer is controversial. ${ }^{16}$ More data on this issue are expected from the IMVIGOR 130 study.

As anticipated, an important difference in ORR was found between the cohort of patients with poor $(12 \%)$ in comparison to good ECOG PS (27\%). Similarly, poor ORR in patients with ECOG PS 2 have been reported in the SAUL trial, which recruited real-world patients with $\mathrm{mUC}$, rarely included in prospective trials. ${ }^{19}$ Previous studies have shown that only a subset of patients with $\mathrm{mUC}$ are able to receive more lines of therapies as their ECOG PS rapidly deteriorates. ${ }^{20}$ Therefore, more research efforts need to be put into optimizing the selection of first-line therapy.

Only one patient with liver metastases responded to atezolizumab 1 (6\%). Liver metastases were associated with reduced marginal CD8 ${ }^{+} \mathrm{T}$-cell infiltration, providing a potential mechanism for this outcome.21 A similar proportion of our patients with poor and good PS by the WHO had liver metastasis (in poor PS 10/26, 28\%, vs. in good PS 8/18, $31 \%$ ) Therefore, the reduced benefit in our population with poor ECOG PS could not be attributed to the site of metastasis, but rather to other uninvestigated factors.

Overall, the mOS of our patient groups was shorter than the mOS of similar patient groups reported in prospective studies. The mOS of our platinum-treated group was shorter in comparison to the intention to treat the population (all platinumtreated) in IMVIGOR 211 or to the platinum-treated cohort 2, in IMVIGOR 210 (6.8 vs. 8.6 and 7.8 months), respectively. An even larger difference in
mOS was observed among our platinum-naive cohort and cisplatinum-ineligible patients in cohort 1 of IMVIGOR 210 and the randomized large group of 451 patients receiving first-line monotherapy with atezolizumab in IMVIGOR 130 (8.7 vs. 15.8 and 16 months), respectively. ${ }^{12,22}$ Very short mOS of only $2.3 \mathrm{~m}$ was reported in patients with poor ECOG PS in the prospective SAUL real-world analysis. ${ }^{19}$ In total, we had $42 \%$ of patients with ECOG PS of two or more, which was four times higher than the proportion of patients with poor ECOG PS represented in the SAUL study population. Almost $30 \%$ of our patients died within the first two months after receiving the first dose of atezolizumab (Figure 2). It was reported that nearly double as many cancer patients initiated systemic therapy near the end of life mainly due to increased immune checkpoint inhibitor (ICI) use. ${ }^{23}$ Possibly medical oncologists do not fear toxicities of chemotherapy and may prescribe ICI even to patients who are not fit or eligible for chemotherapy. ${ }^{24}$

We may conclude that the mOS of our whole group was compromised by a large cohort of patients with poor ECOG PS who died even before the first radiographic evaluation. Of interest is the fact that no consensus could be reached by the ESMO Guidelines Committee on whether immune checkpoint inhibitors could be recommended for firstline therapy of PD-L1 negative patients not eligible for any chemotherapy as stated on an e-update in $2019 .{ }^{25}$ Based on our study, the initiation of secondline systemic therapy with an immune checkpoint inhibitor in patients with poor ECOG PS should be discussed on an individual patient-therapist basis. For the majority of these patients providing best supportive care may be the best option.

Another explanation of a large proportion of patients dying after one or two cycles of atezolizumab may be a potential hyperprogression of the disease. As hyperprogression of cancer by definition needs at least a well-defined course of the disease before initiation of ICI, this hypothesis could not be resolved in a retrospective trial. ${ }^{26}$

We report clinical prognostic factors for OS in platinum-treated patients with mUC receiving an immune checkpoint inhibitor, atezolizumab, in a real-world practice. In univariant analysis three parameters: ECOG PS 1, 0, TFI more than six months, and $\mathrm{CB}$ with chemotherapy, showed a statistically significant correlation with longer survival. Poor ECOG PS has been identified as a poor prognostic factor in other studies with immune checkpoint. ${ }^{23,27}$ Such patients are often excluded from the randomized clinical trials, therefore real-world data 
are important for this population. Poor ECOG PS, however, did not retain the prognostic significance for survival in multivariant analysis in our patient cohort, which may be due to the poor reliability of this score. ${ }^{15,28}$

Furthermore, we found that disease control with chemotherapy was important for the efficiency of the immune checkpoint inhibitor. Disease control parameters, represented by TFI and CB to prior therapy, both showed a prognostic value for survival in univariant analysis. In multivariant analysis only TFI of less than six months retained a negative prognostic value for OS (Table 3). This finding may help in designing future prospective clinical trials. In fact, the JAVELIN 100 phase III trial permitted only patients that achieved $\mathrm{CB}$ with chemotherapy to continue with maintenance therapy with avelumab or best supportive care. The addition of avelumab to best supportive care was associated with a significant $31 \%$ reduction in the risk of death, with the median OS durations of 21.4 and 14.3 months for the avelumab and BSC alone groups, respectively. ${ }^{29}$

Response to cisplatin-based chemotherapy or immunotherapy, especially checkpoint blockade, has been shown to correlate with molecular subtype. ${ }^{30,31}$ Whether these molecular subtypes were shared in patients that were not responsive to either therapy is currently unknown. So far, no known molecular markers showing clinical utility to select patients that are not responsive to cisplatin based chemotherapy or ICI are available. ${ }^{32}$ Moreover, molecular alterations induced by chemotherapy were poorly characterized. Chemotherapy probably plays an important role in enhancing the immunogenicity of tumor, making it more susceptible to therapy with ICI. ${ }^{33}$ Based on the results of IMVIGOR 130 the concomitant effect of chemotherapy and atezolizumab is important as a combination of these two significantly prolonged PFS compared to chemotherapy alone. ${ }^{12}$

Nevertheless, it is important that clinicians foresee which patients have a poor prognosis even with ICI. Such patients need careful monitoring when treated with ICI or should perhaps be offered an alternative treatment, if available, or even best supportive treatment instead.

The rate grade 3-4 of immune adverse events we observed was similar to that reported in the prospective SAUL trial (11 \% vs. 13\%). The latter trial included a wide selection of patients. Due to the retrospective nature of our investigation the grading of AE was not exact, nor has the potential im- mune-related condition been appropriately tested. One of our patients suffered severe neurologic deterioration that we attributed to probable autoimmune encephalitis. The types of immune adverse events we describe (Table 4 ) were already reported elsewhere. ${ }^{12,18}$

The present study has several limitations. First, the number of patients was too low to be able to draw definitive conclusions. Second, the retrospective study design has weak points such as a heterogeneous group of patients, non-consistent timing of radiographic evaluation, unconfirmed reporting of, possibly, irAE and missing data such as PD-L1 testing scores.

\section{Conclusions}

In total, we have confirmed a long-lasting response to atezolizumab in a proportionally similar subset of patients with mUC treated in daily practice comparable to prospective trials. The mOS of all platinum-naive and platinum-treated patients was shorter than the mOS of similar patient groups reported in prospective studies, mainly due to a high percentage of our patients with poor ECOG PS. Significantly, TFI of less than six months is a clinically-important poor prognostic factor for OS. It would be of clinical value to investigate if patients with FGFR mutation or fusion acquiring disease control with chemotherapy benefit more from an immune checkpoint inhibitor or a FGFR inhibitor. In conclusion, whether disease control with chemotherapy is also predictive of an atezolizumab treatment effect, or not, can only be assessed in a valid comparative setting such as in a randomized trial. If it is predictive, then these patients may share a common molecular genetic profile.

\section{Acknowledgments}

We thank Assistant Prof. Janja Jerebic, Ph.D., Faculty of Organizational Sciences, University in Maribor, who performed the statistical analysis

\section{Author contributions}

All authors contributed with the study design, data collection, data analysis, and writing of the manuscript. All authors have read and approved the final manuscript. 


\section{References}

1. Torre LA, Siegel RL, Ward EM, Jemal A. Global Cancer Incidence and Mortality Rates and Trends-An Update. Cancer Epidemiol Biomarkers Prev 2016; 25: 16-27. doi: 10.1158/1055-9965.EPI-15-0578

2. Oing C, Rink M, Oechsle K, Seidel C, von Amsberg G, Bokemeyer C. Second line chemotherapy for advanced and metastatic urothelial carcinoma: vinflunine and beyond - a comprehensive review of the current literature. $J$ Urol 2016; 195: 254-63. doi: 10.1016/j.juro.2015.06.115

3. Galsky MD, Hahn NM, Rosenberg J, Sonpavde G, Hutson T, Oh WK, et al. Treatment of patients with metastatic urothelial cancer "unfit" for cisplatin-based chemotherapy. J Clin Oncol 2011; 29: 2432-8. doi: 10.1200/ JCO.2011.34.8433

4. Bellmunt J, Théodore C, Demkov T, Komyakov B, Sengelov L, Daugaard G, et al. Phase III trial of vinflunine plus best supportive care compared with best supportive care alone after a platinum-containing regimen in patients with advanced transitional cell carcinoma of the urothelial tract. J Clin Oncol 2009; 27: 4454-61. doi: 10.1200/JCO.2008.20.5534

5. Bamias A, Tzannis K, Harshman LC, Crabb SJ, Wong Y-N, Kumar Pal S, et al. Impact of contemporary patterns of chemotherapy utilization on survival in patients with advanced cancer of the urinary tract: a Retrospective International Study of Invasive/Advanced Cancer of the Urothelium (RISC). Ann Oncol 2018; 29: 361-9. doi: 10.1093/annonc/mdx692

6. Apolo AB, Infante JR, Balmanoukian A, Patel MR, Wang D, Kelly K, et al. Avelumab, an anti-programmed death-ligand 1 antibody, in patients with refractory metastatic urothelial carcinoma: results from a multicenter, phase Ib study. J Clin Oncol 2017; 35: 2117-24. doi: 10.1200/JCO.2016.71.6795

7. Massard C, Gordon MS, Sharma S, Rafii S, Wainberg ZA, Luke J, et al. Safety and efficacy of durvalumab (MEDI4736), an anti-programmed cell death ligand-1 immune checkpoint inhibitor, in patients with advanced urothelial bladder cancer. J Clin Oncol 2016; 34: 3119-25. doi: 10.1200/ JCO.2016.67.9761

8. Powles T, Durán I, van der Heijden MS, Loriot Y, Vogelzang NJ, De Giorgi U, et al. Atezolizumab versus chemotherapy in patients with platinum-treated locally advanced or metastatic urothelial carcinoma (IMvigor211): a multicentre, open-label, phase 3 randomised controlled trial. Lancet 2018; 391 748-57. doi: 10.1016/S0140-6736(17)33297-X

9. Sharma P, Retz M, Siefker-Radtke A, Baron A, Necchi A, Bedke J, et al. Nivolumab in metastatic urothelial carcinoma after platinum therapy (CheckMate 275): a multicentre, single-arm, phase 2 trial. Lancet Oncol 2017; 18: 312-22. doi: 10.1016/S1470-2045(17)30065-7

10. Balar AV, Galsky MD, Rosenberg JE, Powles T, Petrylak DP, Bellmunt J, et al Atezolizumab as first-line treatment in cisplatin-ineligible patients with locally advanced and metastatic urothelial carcinoma: a single-arm, multicentre, phase 2 trial. Lancet 2017; 389: 67-76. doi: 10.1016/S0140-6736(16)324552

11. Balar A V, Castellano D, O'Donnell PH, Grivas P, Vuky J, Powles T, et al. Firstline pembrolizumab in cisplatin-ineligible patients with locally advanced and unresectable or metastatic urothelial cancer (KEYNOTE-052): a multicentre single-arm, phase 2 study. Lancet Oncol 2017; 18: 1483-92. doi: 10.1016/ S1470-2045(17)30616-2

12. Galsky MD, Arija JÁA, Bamias A, Davis ID, De Santis M, Kikuchi E, et al. Atezolizumab with or without chemotherapy in metastatic urothelial cance (IMvigor130): a multicentre, randomised, placebo-controlled phase 3 trial. Lancet 2020; 395: 1547-57. doi: 10.1016/S0140-6736(20)30230-0

13. Bellmunt J, Choueiri TK, Fougeray R, Schutz FAB, Salhi $Y$, Winquist $E$, et al. Prognostic factors in patients with advanced transitional cell carcinoma of the urothelial tract experiencing treatment failure with platinum-containing regimens. J Clin Oncol 2010; 28: 1850-5. doi: 10.1200/JCO.2009.25.4599

14. Matsumoto R, Abe T, Ishizaki J, Kikuchi H, Harabayashi T, Minami K, et al. Outcome and prognostic factors in metastatic urothelial carcinoma patients receiving second-line chemotherapy: an analysis of real-world clinical practice data in Japan. Jpn J Clin Oncol 2018; 48: 771-6. doi: 10.1093/jjco/hyy094

15. Ding X, Chen Q, Yang Z, Li J, Zhan H, Lu N, et al. Clinicopathological and prognostic value of PD-L1 in urothelial carcinoma: a meta-analysis. Cancer Manag Res 2019; 11: 4171-84. doi: 10.2147/CMAR.S176937

16. Rouanne M, Radulescu C, Adam J, Allory Y. PD-L1 testing in urothelia bladder cancer: essentials of clinical practice. World J Urol 2020; [Ahead of print]. doi: 10.1007/s00345-020-03498-0
17. Rosenberg JE, Hoffman-Censits J, Powles T, Van Der Heijden MS, Balar A V., Necchi A, et al. Atezolizumab in patients with locally advanced and metastatic urothelial carcinoma who have progressed following treatment with platinum-based chemotherapy: a single-arm, multicentre, phase 2 trial. Lancet 2016; 387: 1909-20. doi: 10.1016/S0140-6736(16)00561-4

18. Stewart TF, Petrylak DP. Atezolizumab in understudied populations with bladder cancer. Nat Rev Urol 2019; 16: 449-50. doi: 10.1038/s41585-0190200-8

19. Sternberg CN, Loriot $\mathrm{Y}$, James N, Choy E, Castellano D, Lopez-Rios F, et al. Primary results from SAUL, a multinational single-arm safety study of atezolizumab therapy for locally advanced or metastatic urothelial or nonurothelial carcinoma of the urinary tract. Eur Urol 2019; 76: 73-81. doi: 10.1016/j.eururo.2019.03.015

20. Flannery K, Boyd M, Black-Shinn J, Robert N, Kamat AM. Outcomes in patients with metastatic bladder cancer in the USA: a retrospective electronic medical record study. Future Oncol 2019; 15: 1323-34. doi: 10.2217/fon2018-0654

21. Tumeh PC, Hellmann MD, Hamid O, Tsai KK, Loo KL, Gubens MA, et al. Liver metastasis and treatment outcome with anti-PD-1 monoclonal antibody in patients with melanoma and NSCLC. Cancer Immunol Res 2017; 5: 417-24. doi: $10.1158 / 2326-6066 . C I R-16-0325$

22. Rosenberg J, Petrylak D, Abidoye O, Van der Heijden MS, Hofman-Censits J, Necchi A, et al. 21LBA Atezolizumab in patients (pts) with locally-advanced or metastatic urothelial carcinoma (mUC): results from a pivotal multicente phase II study (IMvigor 210). Eur J Cancer 2015; 51(Suppl 3): S720. [abstract]. doi: 10.1016/s0959-8049(16)31942-6

23. Parikh RB, Galsky MD, Gyawali B, Riaz F, Kaufmann TL, Cohen AB, et al. Trends in checkpoint inhibitor therapy for advanced urothelial cell carcinoma at the end of life: insights from real-world practice. Oncologist 2019; 24: e397-9. doi: 10.1634/theoncologist.2019-0039

24. Schnipper LE, Smith TJ, Raghavan D, Blayney DW, Ganz PA, Mulvey TM, et al. American Society of Clinical Oncology identifies five key opportunities to improve care and reduce costs: the top five list for oncology. J Clin Oncol 2012; 30: 1715-24. doi: 10.1200/JCO.2012.42.8375

25. Bellmunt J, Orsola A, Leow JJ, Wiegel T, De Santis M, Horwich A. Bladder cancer: ESMO practice guidelines for diagnosis, treatment and follow-up. Ann Oncol 2014; 25: iii40-8. doi: 10.1093/annonc/mdu223

26. Frelaut M, Le Tourneau C, Borcoman E. Hyperprogression under immunotherapy. Int J Mol Sci 2019; 20: 2674. doi: 10.3390/ijms20112674

27. Khaki AR, Li A, Diamantopoulos LN, Bilen MA, Santos V, Esther J, et al. Impact of performance status on treatment outcomes: a real-world study of advanced urothelial cancer treated with immune checkpoint inhibitors. Cancer 2020; 126: 1208-16. doi: 10.1002/cncr.32645

28. Sørensen JB, Klee M, Palshof T, Hansen HH. Performance status assessment in cancer patients. an inter-observer variability study. Br J Cancer 1993; 67: 773-5. doi: 10.1038/bjc.1993.140

29. Powles T, Park SH, Voog E, Caserta C, Valderrama BP, Gurney H, et al. Avelumab maintenance therapy for advanced or metastatic urothelial carcinoma. N Engl J Med 2020; 383: 1218-30. doi: 10.1056/nejmoa2002788

30. Choi W, Porten S, Kim S, Willis D, Plimack ER, Hoffman-Censits J, et al. Identification of distinct basal and luminal subtypes of muscle-invasive bladder cancer with different sensitivities to frontline chemotherapy. Cancer Cell 2014; 25: 152-65. doi: 10.1016/j.ccr.2014.01.009

31. Seiler R, Ashab HAD, Erho N, van Rhijn BWG, Winters B, Douglas J, et al. Impact of molecular subtypes in muscle-invasive bladder cancer on predicting response and survival after neoadjuvant chemotherapy. Eur Urol 2017; 72: 544-54. doi: 10.1016/j.eururo.2017.03.030

32. Buttigliero C, Tucci M, Vignani F, Scagliotti GV, Di Maio M. Molecular biomarkers to predict response to neoadjuvant chemotherapy for bladder cancer. Cancer Treat Rev 2017; 54: 1-9. doi: 10.1016/j.ctrv.2017.01.002

33. Luo $Q$, Zhang L, Luo $C$, Jiang $M$. Emerging strategies in cancer therapy combining chemotherapy with immunotherapy. Cancer Lett 2019; 454: 191-203. doi: 10.1016/j.canlet.2019.04.017 